\title{
Characterization of the SPI-1 and Rsp type three secretion systems in Pseudomonas fluorescens F113
}

\author{
Matthieu Barret,, ${ }^{1,2}$ Frank Egan,, \\ Jennifer Moynihan, ${ }^{1,2}$ John P. Morrissey, ${ }^{2}$ \\ Olivier Lesouhaitier ${ }^{3}$ and Fergal O'Gara, ${ }^{1,2 *}$ \\ ${ }^{1}$ BIOMERIT Research Centre, and ${ }^{2}$ Department of \\ Microbiology, University College Cork, Cork, Ireland. \\ ${ }^{3}$ Normandie University, University of Rouen, LMSM, EA \\ 4312, F-27000 Evreux, France.
}

\section{Summary}

Pseudomonas fluorescens F113 is a plant growthpromoting rhizobacterium (PGPR) isolated from the sugar beet rhizosphere. The recent annotation of the F113 genome sequence has revealed that this strain encodes a wide array of secretion systems, including two complete type three secretion systems (T3SSs) belonging to the Hrp1 and SPI-1 families. While Hrp1 T3SSs are frequently encoded in other $\boldsymbol{P}$. fluorescens strains, the presence of a SPI-1 T3SS in a plantbeneficial bacterial strain was unexpected. In this work, the genetic organization and expression of these two T3SS loci have been analysed by a combination of transcriptional reporter fusions and transcriptome analyses. Overexpression of two transcriptional activators has shown a number of genes encoding putative T3 effectors. In addition, the influence of these two T3SSs during the interaction of $P$. fluorescens F113 with some bacterial predators was also assessed. Our data revealed that the transcriptional activator hilA is induced by amoeba and that the SPI-1 T3SS could potentially be involved in resistance to amoeboid grazing.

\section{Introduction}

Non-flagellar type III secretion systems (T3SS) are nanomachines composed of approximately 25 proteins, encoded in Gram-stain-negative bacteria belonging to the Proteobacteria, Chlamydia and Verrucomicrobia phyla (Cornelis, 2010; Izoré et al., 2011; Sait et al., 2011; Barret et al., 2013). T3SSs are specialized machineries dedicated to the translocation of a wide diversity of Type III

Received 1 November, 2012; accepted 10 January, 2013. *For correspondence. E-mail f.ogara@ucc.ie; Tel. (+353) (0)21 427 2646; Fax (+353) (0)21 4275934 effectors (T3Es) from the bacterial cytosol to the eukaryotic host cytoplasm (Dean, 2011). These T3Es could be involved in numerous types of bacterial-host interactions ranging from mutualism to parasitism (Preston, 2007; Coombes, 2009). In contrast to the large diversity observed among T3S effectors, the structural apparatus (or injectisome) itself is highly conserved and has evolved into seven different phylogenetic families: Ysc, Hrp1, Hrp2, SPI-1, SPI-2, Rhizobial and Chlamydial (Pallen et al., 2005; Troisfontaines and Cornelis, 2005; Abby and Rocha, 2012).

Modulation of host metabolism by T3Es has been initially studied in plant, animal or human pathogens. However, in the last decade the influence of T3SSs in rhizosphere colonization by plant beneficial bacteria has been investigated in a number of studies. For instance, the protein-coding gene $r s c C$ involved in the assembly of the Hrp1-like T3SS apparatus of the biocontrol strain Pseudomonas fluorescens SBW25 has been shown to be induced in the sugar beet rhizosphere (Rainey, 1999). Subsequent analysis has revealed that the Hrp1-like T3SS of P. fluorescens SBW25 (also designated Rsp/ Rsc) was able to elicit a hypersensitive response in Nicotiana clevelandii, proving its functionality (Preston et al., 2001). Hrp1 genes were found to be widespread among a range of plant growth-promoting bacteria (PGPR) belonging to the P. fluorescens group (Preston et al., 2001; Mazurier et al., 2004; Rezzonico et al., 2004; Viollet et al., 2011). In addition, numerous Hrp1 loci were found in whole or partial genome sequences of strains related to the P. fluorescens group (Rezzonico et al., 2005; Kimbrel et al., 2010; Cusano et al., 2011; Mavrodi et al., 2011; Ortet et al., 2011; Loper et al., 2012). The abundance of Hrp1 T3SSs in genomes of strains related to the P. fluorescens group, coupled with its expression in different rhizospheres (Jackson et al., 2005; Rezzonico et al., 2005; Mavrodi et al., 2011) implies its ecological significance during 'beneficial plant-bacterial interactions'. Recent evidence suggests that Hrp1 of $P$. brassicacearum Q8r1-96 could be involved in suppression of PAMPtriggered immunity (PTI) through translocation of T3Es related to $P$. syringae strains (Mavrodi et al., 2011). Suppression of $\mathrm{PTI}$ by T3Es could facilitate colonization of root tissues by Hrp1 positive strains, or alternatively, improve the establishment of ectomycorrhizal symbiosis, a phenomenon observed with the Hrp1 T3SS of the 
mycorrhiza helper bacteria P. fluorescens BBc6R8 (Cusano et al., 2011). However, these findings are unlikely to be transposed to all Hrp1 positive strains related to the $P$. fluorescens group since the Hrp1 locus and, more importantly the T3E repertoire, vary considerably between strains.

Pseudomonas fluorescens F113 is a PGPR strain isolated from the sugar-beet rhizosphere in Ireland (Shanahan et al., 1992). Initially this strain was selected and studied because of its capacity to inhibit growth of a range of phytopathogenic bacteria, fungi, oomycetes and nematodes including Pectobacterium carotovorum (Cronin et al., 1997b), Fusarium oxysporum (Barahona et al., 2011), Pythium ultimum (Fenton et al., 1992) and Globodera spp. (Cronin et al., 1997a). This antimicrobial capacity is strongly linked to the production of a secondary metabolite, 2,4-diacetylphloroglucinol (Fenton et al., 1992; Abbas et al., 2002). In addition, P. fluorescens F113 is also an excellent rhizosphere colonizer of different plant species including wheat (De La Fuente et al., 2006), alfalfa (Villacieros et al., 2003), and willow (de Carcer et al., 2007). For these reasons, F113 is a widely used model strain for studying rhizosphere colonization (Martínez-Granero et al., 2006; Barahona et al., 2010).

The sequencing of the $P$. fluorescens $\mathrm{F} 113$ genome has confirmed the presence of a complete Hrp1 locus (Preston et al., 2001), but has also revealed the existence of a T3SS locus related to the SPI-1 family (RedondoNieto et al., 2012). SPI-1 T3SSs have been mostly found in bacteria associated with mammals (Galan, 1999; Miki et al., 2010). However, recent sequencing projects have also identified SPI-1 T3SSs in chromosomes of plant pathogenic bacteria such as Xanthomonas albilineans (Marguerettaz et al., 2011) and Pantoea stewartii (Correa et al., 2012). Moreover, some bacterial strains related to the $P$. fluorescens group like P. fluorescens HK44 (Chauhan et al., 2011) and P. fluorescens Q2-87 (Loper et al., 2012) also possess the SPI- 1 cluster. The presence of SPI-1 T3SSs in commensal rhizospheric bacterial strains raises several questions. First, whether this system is expressed at all and under which conditions it is induced, and then what is its role in the rhizocompetence of these bacterial strains.

In this work two transcriptional activators of Hrp1 and $\mathrm{SPI}-1$ have been identified in $P$. fluorescens F113. Following transcriptome comparisons, a number of $\mathrm{F} 113$ genes induced by these two regulators were highlighted. These included genes encoding protein involved in the assembly of the structural apparatus and putative T3Es. In addition, the potential roles of these T3SSs during interactions between $\mathrm{F} 113$ and other soil organisms were assessed. Our results demonstrate that the SPI-1 T3SS is induced during F113-amoeba interactions and is involved in resistance to amoeboid predation.

\section{Results and discussion}

Genetic organization of the SPI-1 T3SS

in Pseudomonas

Comparative genomic analysis of the SPI-I clusters encoded in the strains belonging to the $P$. fluorescens group (Figs $1 \mathrm{~A}$ and $\mathrm{S} 1$ ) indicates a predominantly conserved genetic organization (Fig. 1B). Indeed, all the protein coding genes potentially involved in the assembly of the export apparatus (spaL, spaP, spaQ, spaR, spaS and invA), the basal body (prgH, prgJ, prjK and invG), the needle ( $p r j)$ and the gene encoding a class II chaperone $(\operatorname{sic} A)$ display more than $60 \%$ identity to each other at the amino acid level. However, protein-coding genes involved in the assembly of the cytoplasmic sorting platform (spaO, $\operatorname{org} A$ and $\operatorname{org} B)$ and the putative translocon $(\operatorname{sip} B, \operatorname{sip} C$ and $\operatorname{sip} D$ ) are less conserved across the strains related to the $P$. fluorescens group (between 40 and $80 \%$ identity). In addition, some variations exist between the $P$. fluorescens $\mathrm{SPI}-1$ clusters. For example, the gene invB encoding a putative class IB chaperone (Ehrbar et al., 2003) in P. fluorescens HK44 and Pseudomonas sp. GM49 is not found in SPI- 1 clusters of $P$. fluorescens $\mathrm{F} 113$ and Q2-87, which could indicate the presence of different T3E repertoires between these strains. Finally, some proteins exhibit low sequence conservation to proteins generally encoded in other SPI-1 clusters from different bacterial species. For instance, the protein-coding gene PSF113_1783 located in place of invJ in the SPI-1 cluster of Salmonella displays no substantial sequence identity at the amino acid level with InvJ. Since InvJ is involved in controlling needle length (Cornelis, 2006), this might mean that the mechanism for controlling needle length may be different in $P$. fluorescens.

\section{RspL and HilA are transcriptional activators of Hrp1 and SPI-1 genes}

Previous studies have shown that the transcriptional activator HilA and the alternative sigma factor RspL induce expression of genes located in the SPI-1 and Hrp1 clusters respectively (Bajaj et al., 1995; Jackson et al., 2005). To see if these findings were transposable to the SPI-1 and Hrp1 clusters of $P$. fluorescens F113, strains that constitutively expressed either hilA (PSF113_1794) or $r s p L$ (PSF113_5610) were engineered by cloning these genes in the broad host range vector pBBR1MCS5 (Kovach et al., 1995) (details on plasmid and mutants constructions are indicated in the experimental procedures provided in Supporting information). According to comparative transcriptomic analyses constitutive expression of hilA and $r s p L$ lead to slight transcriptome changes in $\mathrm{F} 113$, with 71 (approximately $1 \%$ of the whole predicted transcriptome) and $141(2 \%)$ genes 
A

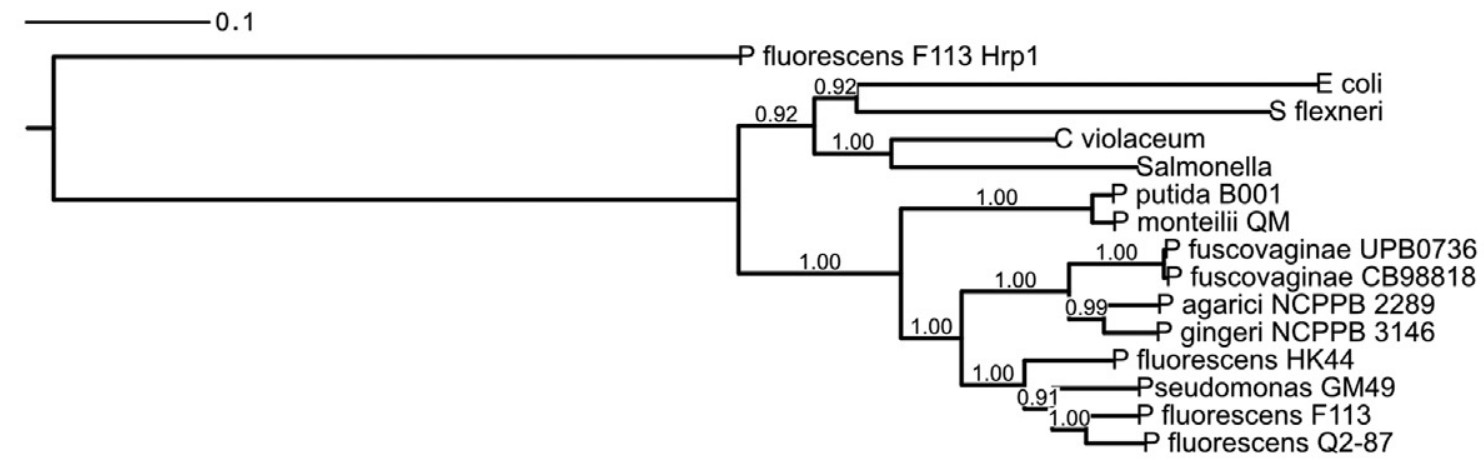

B P. fluorescens F113

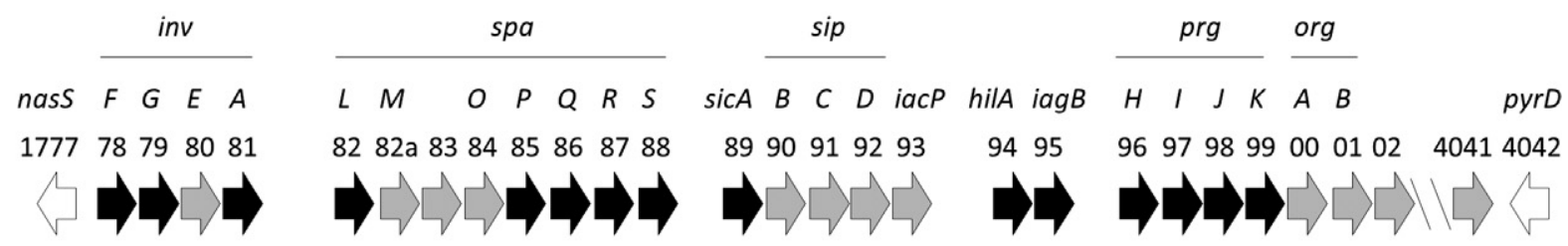

Pseudomonas sp. GM49

$\operatorname{inv} B$

$2359606102707172 \quad 7374757677787980 \quad 8182838485 \quad 8687 \quad 88899091929394 \quad 9596$

P. fuscovaginae UPB0736

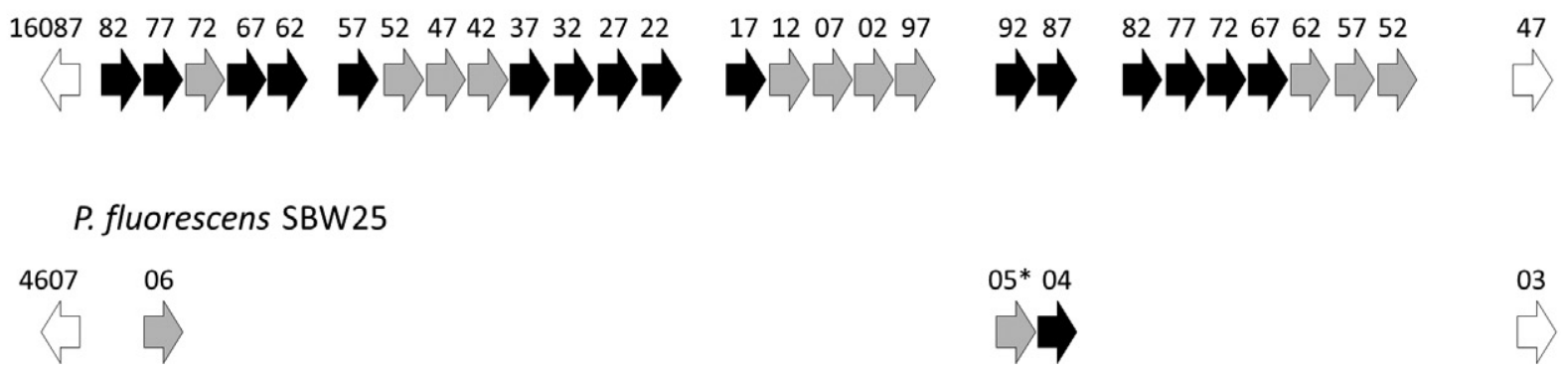

Fig. 1. Analysis of the SPI-1 cluster of different bacterial strains related to the P. fluorescens group.

A. Phylogenetic distribution of SPI-1 clusters. A distance tree (Maximum likelihood) was calculated from InvA homologues. Only aLRT support values greater than 0.75 (1000 replicates) are displayed.

B. Comparative genomic analysis of the SPI-1 T3SS clusters. Genes encoding T3SSs are represented as block arrows showing the direction of their transcription. Numbers represent the locus identifiers. White arrows represent genes encoding non-T3SS proteins. Grey and black arrows represent T3SS and T3E-related protein-coding genes with less or more than $60 \%$ identity at the amino acid level respectively. Asterisks indicate pseudogenes. The SPI-1 cluster of GM49 is located on two separate scaffolds.

(C) 2013 John Wiley \& Sons Ltd and Society for Applied Microbiology, Environmental Microbiology Reports, 5, 377-386 
Table 1. Microarray data validation by qRT-PCR.

\begin{tabular}{|c|c|c|c|c|c|c|}
\hline \multirow[b]{2}{*}{ Locus } & \multirow[b]{2}{*}{ Gene name } & \multirow[b]{2}{*}{ Function } & \multicolumn{2}{|c|}{ HilA/MCS5 (log2) } & \multicolumn{2}{|c|}{ RspL/MCS5 (log2) } \\
\hline & & & Array & qRT-PCR & Array & qRT-PCR \\
\hline PSF113_0435 & & Chitin binding protein & -2.3 & $-0.7 \pm 0.6$ & -1.3 & n.d. \\
\hline PSF113_1783 & & Type III secretion protein & 6.5 & $6.4 \pm 0.3$ & - & n.d. \\
\hline PSF113_1794 & hilA & Type III secretion transcriptional activator HilA & 6.1 & $10.6 \pm 0.4$ & - & n.d. \\
\hline PSF113_1799 & $\operatorname{prgK}$ & Type III secretion protein, PrgK & 5.9 & $8.2 \pm 0.3$ & - & n.d. \\
\hline PSF113_1802 & & Putative Type III effector & 7.0 & $7.1 \pm 0.2$ & - & n.d. \\
\hline PSF113_4041 & & Putative Type III effector & 5.3 & $6.3 \pm 0.2$ & - & n.d. \\
\hline PSF113_3047 & & Non-ribosomal peptide synthetase & 1.5 & $1.6 \pm 0.5$ & - & n.d. \\
\hline PSF113_4698 & & Transcriptional regulator, $\mathrm{PadR}$ family & 6.0 & $5.6 \pm 0.3$ & - & n.d. \\
\hline PSF113_1126 & $\operatorname{rop} A A 1$ & Type III effector RopAA1 & - & n.d. & 3.3 & $4.5 \pm 0.5$ \\
\hline PSF113_3486 & rорAA2 & Putative Type III effector RopAA2 & - & n.d. & - & $0.2 \pm 0.1$ \\
\hline PSF113_5609 & rspJ & Type III secretion protein RspJ & - & n.d. & 3.9 & $5.1 \pm 0.6$ \\
\hline PSF113_5610 & $r s p L$ & RNA polymerase sigma-70 factor, ECF subfamily, RspL & - & n.d. & 9.6 & $9.9 \pm 0.2$ \\
\hline PSF113_5616 & ropM & Type III effector RopM & - & n.d. & 4.0 & $3.6 \pm 0.2$ \\
\hline PSF113_1175 & & Baseplate assembly protein $\mathrm{J}$ & - & n.d. & -2.1 & $-0.7 \pm 0.7$ \\
\hline PSF113_4295 & & Phage tail fibre protein & - & n.d. & -8.0 & $-12.7 \pm 1.4$ \\
\hline PSF113_1757 & pvdH & Pyoverdin biosynthesis protein $\mathrm{PvdH}$ & - & n.d. & -1.8 & $0.1 \pm 0.5$ \\
\hline
\end{tabular}

Microarray data presented here represent only one replicate for each strain. Only $\log _{2}$ ratios inferior to -1 and superior to 1 are shown in this table. Real-time PCR analyses were performed on some selected differentially expressed genes. Averages and associated standard deviations were calculated from three independent biological replicates, each having three technical replicates. All the values have been transformed to $\log _{2}$.

differentially regulated in comparison to the strain carrying the empty vector (Fig. S2). Since microarray data presented in this paper represent only one replicate for each strain, selected genes were validated by qRT-PCR on three independent biological replicates.

The transcriptional activator HilA positively regulates the expression of 51 genes, 26 of which are SPI-1 related (Tables 1 and S1). Among these 26 genes, 24 encode regulatory elements or proteins involved in the assembly of the T3SS and two (PSF113_1802 and PSF113_4041) encode proteins with $\mathrm{T} 3$ secretion signals. RspL positively regulates the expression of 41 genes, 29 of which are Hrp1 related (Tables 1 and S1). This includes all the genes encoded in the Hrp1 locus with the exception of $r s p S$. This is not surprising since RspS orthologues act upstream of RspL orthologues in the regulatory cascades described in P. syringae and Dickeya sp. (Hutcheson et al., 2001; Tang et al., 2006). Whereas the putative T3Es $\operatorname{rop} A A 1$, ropB and ropM are induced by RspL, ropAA2 is not differentially regulated. In addition to protein-coding genes related to Hrp1, RspL downregulates the expression of 40 genes located in a genomic region (PSF113_4278-4325), which is related to prophage 03 of of P. protegens Pf-5 (Mavrodi et al., 2009). The reason for this downregulation is unknown, but interestingly, PrtB, the regulator of pyocin synthesis, is involved in the repression of the Ysc T3SS of P. aeruginosa PAO1 under the stress of DNA damage (Wu and Jin, 2005). Therefore, it is tempting to speculate that T3SSs and prophage elements are inversely regulated in Pseudomonas.
Twenty-one putative RspL binding sites (Mavrodi et al., 2011) were identified, eight of which are located in intergenic regions (Table S2). The predicted promoter regions of these eight genes/operons were aligned and used to generate a consensus F113 RspL binding site (Fig. 2A). This consensus sequence 5'-YGGAAC- $\mathrm{N}_{15-16}$-YCAC$\mathrm{N}_{2}-\mathrm{A}-3^{\prime}$ is slightly different from the initial $h r p$ box 5'-KGGARCY-N ${ }_{15-16}$-CCAC- $\mathrm{N}_{2}-\mathrm{A}-\mathrm{3}^{\prime}$ described in P. syringae (Zwiesler-Vollick et al., 2002). Among the eight promoters possessing an rsp box, six are located upstream of genes upregulated in F113 pBBR-rspL (Table S2). Interestingly, the promoter located upstream of ropAA2 possesses a divergent rsp box (Fig. 3A), which might explain why the expression of ropAA2 is not induced in the strain F113 pBBR1MCS-rspL. Although a HilA binding site consisting of two hexamers separated by five nucleotides has been described in Salmonella typhimurium (Lostroh et al., 2000) and Escherichia coli 042 (Sheikh et al., 2006), no site exhibiting this consensus nucleotide sequence was found in the predicted promoters of SPI-1 genes of $P$. fluorescens $\mathrm{F} 113$.

\section{GacA regulates hilA expression}

The expression of hilA and $r s p L$ were investigated in different synthetic media using promoter gene fusions. According to these promoter fusion assays, $r s p L$ is not expressed in Luria-Bertani, while a basal level of expression is observed for hilA (Fig. 3B and C). Addition of $10 \mathrm{mM}$ of nitrilotriacetic acid, a di and tri metal cation chelator frequently used for induction of Ysc-T3SS gene 


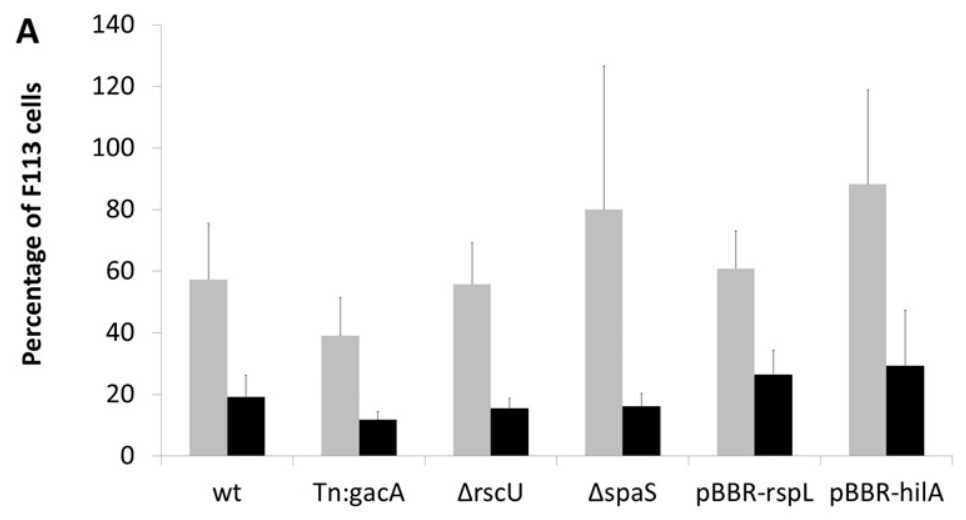

Fig. 2. A. polyphaga-P. fluorescens $\mathrm{F} 113$ interactions.

A. Survival of F113 strains (expressed as percentage) after $24 \mathrm{~h}$ (grey bars) and $48 \mathrm{~h}$ of co-culture with $A$. polyphaga. Values are mean of three biological replicates. Error bars represent standard errors of the means. B. Relative fitness of mutant in PAS media. C. Relative fitness of mutant in PAS media supplemented with amoeba. Fitness is expressed as ratio ( $\log _{10}$ transformed) between strains carrying the vector pBBR1MCS4 and strains carrying the vector pBBR1MCS5. Experiments have been performed on four independent replicates.

B

$1, \mathrm{E}+01$

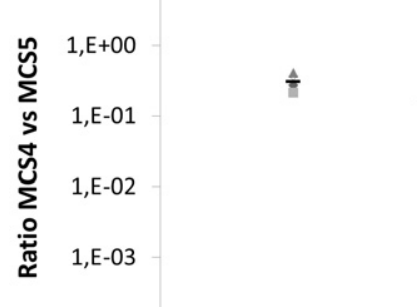

$1, \mathrm{E}-04$

C

$1, \mathrm{E}+01$

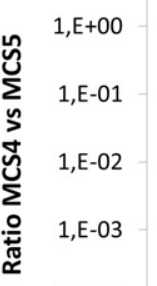

$1, \mathrm{E}-04$

pBBR1MCS4

F113

pBBR1MCS5

F113

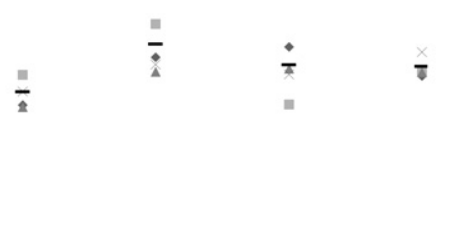

Fis
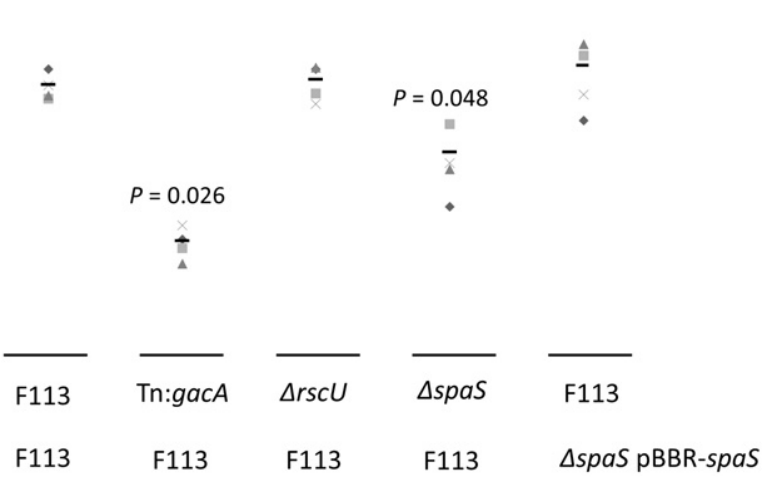

expression in $P$. aeruginosa (Rietsch and Mekalanos, 2006), did not increase $r s p L$ nor hilA promoter activities (Fig. S3). Furthermore, no expression of hilA and rspL was observed in hrp-inducing media (Huynh et al., 1989) (data not shown). Since the GacA orthologue of Salmonella enterica, SirA, positively regulates hilA expression (Altier et al., 2000), the hilA promoter fusion was introduced into the F113 gacA mutant strain FG9 (Delany, 1999; Aarons et al., 2000). In LB and LB NTA media, a decrease in hilA promoter activity was observed in FG9 in comparison to $\mathrm{F} 113$ (Figs $3 \mathrm{C}$ and S3). This suggests that GacA is also a positive regulator of hilA in $P$. fluorescens F113.

The SPI-1 T3SS of P. fluorescens F113 is involved in grazing resistance

To assess the potential role(s) of SPI-1 and Hrp1 T3SSs in interactions with organisms present in the rhizosphere, in-frame deletions of the spaS and $h r c U$ genes were generated. Indeed mutations of spaS and hrcU homologues abolish the secretion of needle and translocator subunits, resulting in non-functional T3SSs (reviewed in Izoré et al., 2011). The effect of both T3SSs of $P$. fluorescens F113 on the mortality of Caenorhabditis elegans was first tested. Under slow-killing conditions (Tan et al., 1999), the gacA negative strain FG9 is severely defective in its ability to kill 
A

\begin{tabular}{ll}
\hline PSF113_0048 & CGGAACCATCACCGCCACACCC-TCACTAA \\
ropAA1 & CGGAACCGATAGGAAAGAGGTACCACACA \\
rspA & TGGAACCGTTAAGCAACGGTCGCCACTTA \\
ropB & TGGAACCGATGCACACGCGCTTGCCACTCA \\
rspF & CGGAACCGCCAACGGCATAACCACCACTCA \\
rspJ & TGGAACTGAATGCTGGATCGCTGCCACCCA \\
rspH & TGGAACCGCAGCCCTGTGATCTGCCACCCA \\
srcM & TGGAACCGAAATGCCGCAGGTG-TCACTCA \\
rsp F113 & YGGAACY--------------YCAC--A \\
hrpDC3000 & KGGARCY--------------CCAC--A \\
ropAA2 & TGGAACGCTTTTTCCGGCACGG-TCACTCA
\end{tabular}
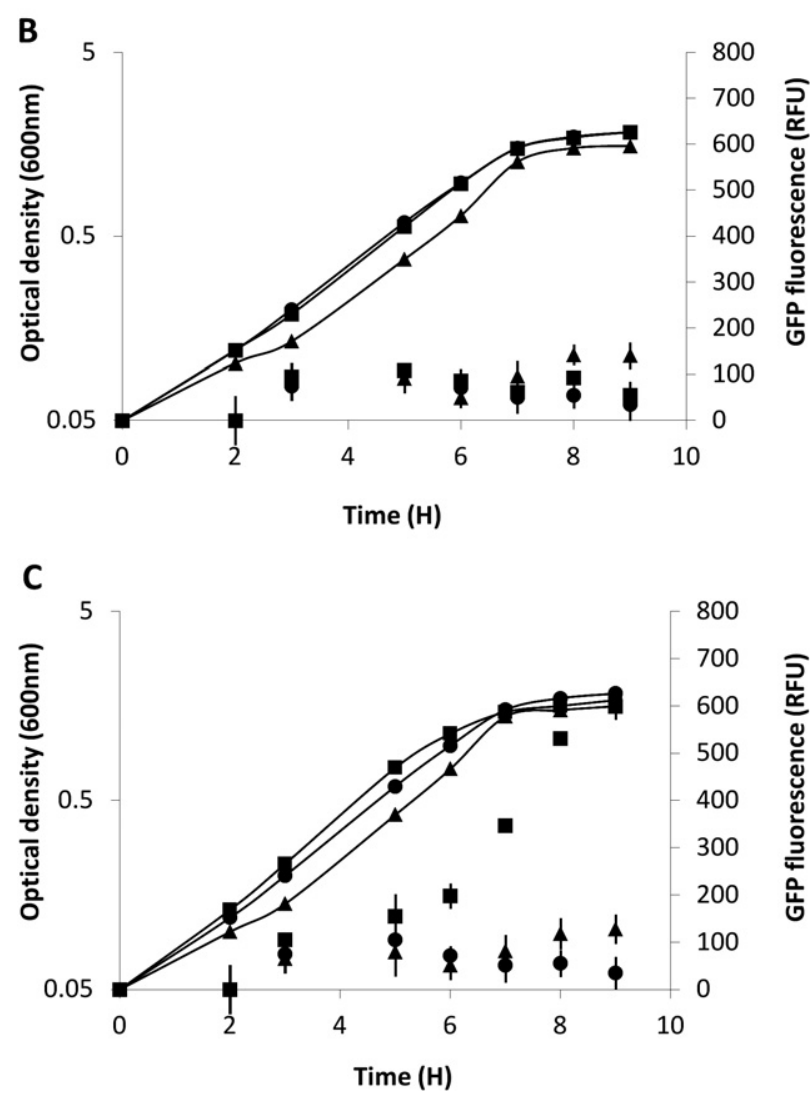

Fig. 3. Expression of hilA and $r s p L$ in synthetic media. A. Consensus ' $r s p$ box' sequence in $P$. fluorescens F113. The putative RspL binding sites of eight genes/operons were aligned using the CLUSTALW program. A consensus sequence (Rsp_F113) is indicated at the bottom of the alignment. This consensus sequence is slightly different from the Hrp box of DC3000

(Zwiesler-Vollick et al., 2002). The putative promoter of ropAA2 potentially contains a divergent RspL binding site.

B. Growth curves (principal $y$ axis) and relative fluorescence units (secondary $y$ axis) were monitored in F113 pPROBE-GT (circle), F113 pPROBE-rspL (square) and in FG9 pPROBE-rspL (triangle). C. The same assay was performed with F113 pPROBE-GT (circle), F113 pPROBE-hilA (square) and in FG9 pPROBE-hilA (triangle). The strain FG9 is a F113 derivative carrying a transposon insertion in gacA (Delany, 1999). Strains of $P$. fluorescens F113 carrying $r s p L(B)$ and hilA (C) transcriptional fusions were grown in LB at $30^{\circ} \mathrm{C}$ shaking. Each value is the mean of three samples obtained from three independent experiments. Error bars represent standard errors of the means.
C. elegans, confirming previous results obtained with P. protegens CHAO (Neidig et al., 2011) (Fig. S4A). In addition, worms exposed to the spaS mutant exhibited an increase in mortality rate relative to control worms feeding on $P$. fluorescens F113 $(P<0.0002)$ (Fig. S4B). The trans-complemented strain presented a virulence activity comparable to the wild type. Although, the same effect is observed for the $r s c U$ mutant, this phenotype is not restored by trans-complementation (Fig. S4C). The increase in the mortality rate of $C$. elegans exposed to spaS mutants could be explained by abolition of SPI-1 T3Es translocation and subsequent inhibition of defensive reaction in C. elegans.

Previous studies have shown that different T3SS families can target a range of unicellular amoebae such as Dictyostelium discoideum and Acanthamoeba castellanii (Pukatzki et al., 2002; Matz et al., 2011; Sperandio et al., 2012). Since soil bacteria are naturally consumed by protozoan predators (Rosenberg et al., 2009), the potential influence of Hrp1 and SPI-1 was assessed during $P$. fluorescens F113-Acanthamoeba polyphaga interactions. To test whether $P$. fluorescens $\mathrm{F} 113$ was susceptible to grazing, A. polyphaga cells were cocultivated with different $\mathrm{F} 113$ strains. After 1 and 2 days of co-culture the relative survival rate of $\mathrm{F} 113$ is around $60 \%$ and $20 \%$ respectively (Fig. $2 \mathrm{~A}$ ). This survival rate is neither significantly different for strains mutated in the gacA, $r s c U$ and spaS genes nor for strains overexpressing hilA and $r s p L$. The result obtained with gacA mutant is somewhat surprising since gacS mutants of $P$. protegens $\mathrm{CHAO}$ are preferentially grazed by $A$. castellanii (Jousset et al., 2009). A selective feeding assay, in which two bacterial strains were co-inoculated as a mixed population with $A$. polyphaga, was then performed. The bacterial strains were inoculated at a ratio of 1:1 in PAS with or without A. polyphaga (Fig. 2B and C). In order to distinguish strains in mixed treatments, the empty vectors pBBR1MCS4 and pBBR1MCS5 were introduced in the different bacterial strains. After $48 \mathrm{~h}$ of interaction no difference in fitness was observed between $\mathrm{F} 113$ and its isogenic mutants when the bacterial strains were grown in PAS (Fig. 2B). However, when amoebae were present in the media the fitness of the gacA mutant was drastically reduced (around 100fold) in comparison with the wild type (Fig. 2C), confirming previous findings obtained with gacS mutant (Jousset et al., 2009). Interestingly, a decrease of fitness was also observed for spaS mutant (approximately 25 -fold) and was trans-complementable by introduction of spaS in the vector pBBR1MCS5 (Fig. 2C). This suggests that the SPI-1 T3SS of $P$. fluorescens $\mathrm{F} 113$ might be involved in resistance to grazing. According to our data, the T3SS-mediated resistance of $\mathrm{F} 113$ to predation is likely to be efficient only in mixed bacterial population, 
A

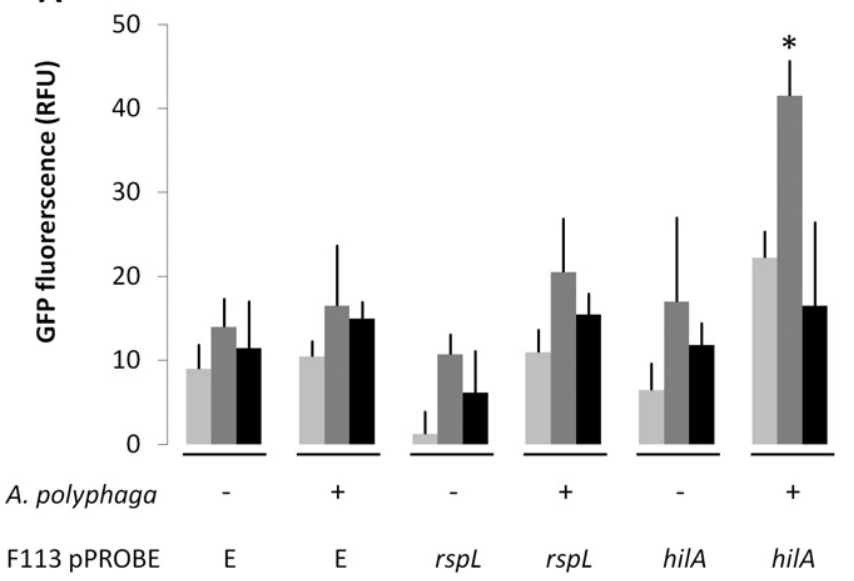

B pMS402

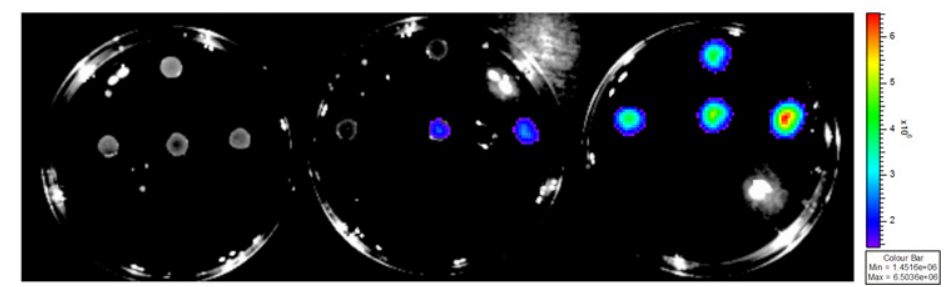

Fig. 4. Induction of hilA by A. polyphaga. A. Promoter activity of hilA during F113A. polyphaga interaction. Expression of $g f p$ reporter fusions were monitored from aliquots of $200 \mu \mathrm{l}$ harvested directly from wells at different time points. Light grey, dark grey and black bars represented 6,24 and $48 \mathrm{~h}$ time-points respectively. Values are means of three biological replicates. Error bars represent standard errors of the means. Asterisks indicate a significant difference in relative fluorescence unit according to unpaired student $t$-test $(P$-value $<0.05)$. B. Composite photographic and luminescence images of hilA and iglA3 promoter fusions. Pseudomonas fluorescens strains carrying the promoterless vector pMS402, pMS-hilA or pMS-iglA3 were spot-inoculated on PYG medium supplemented with trimethoprim 1000. Four spots corresponding to $F 113$ strains without (upper spot) or with amoebae inoculated at a distance of $1 \mathrm{~cm}$ (left), $0.5 \mathrm{~cm}$ (centre) or in contact (right) with the bacterial spot are presented on each plate. Pictures were taken after $48 \mathrm{~h}$ of interaction. The log scales on the right of each image represent luminescence in photons per second per square centimetre per steradian. such as those found in soil, where SPI-I negative bacteria may be preferentially consumed. This phenomenon is similar to the preferential feeding of bacteriovorus amoebae and nematodes on non-toxic gacs mutants (Jousset et al., 2009; 2010).

The effect of $A$. polyphaga on the expression of the T3SS transcriptional activators hilA and $r s p L$ was tested using promoter gene fusions. Under the same conditions used for grazing experiments, the activity of the hilA promoter is significantly increased in strains inoculated in PAS supplemented with A. polyphaga after $24 \mathrm{~h}$ of interaction (Fig. 4A). To confirm this finding, a different bioassay was performed with another reporter gene system based on luminescence. In this assay the relative amount of luminescence of $\mathrm{F} 113$ strains carrying the empty vector pMS402, pMS-hilA or pMS-iglA3 was compared on a PYG media when amoebae were inoculated at a distance of $1 \mathrm{~cm}, 0.5 \mathrm{~cm}$ or in contact with spotinoculated bacteria. The promoter of the T6SS structural gene iglA3, which belongs to the HSI-3 locus of $P$. fluorescens $\mathrm{F} 113$ (Barret et al., 2011) was chosen as a positive control of luminescence since expression of this gene is relatively high under in vitro conditions ( $\mathrm{F}$. Egan, $\mathrm{M}$. Barret and F. O'Gara, unpubl. data). After $48 \mathrm{~h}$ luminescence signals were registered using the IVIS 100 Imaging System. While iglA3 promoter activity is constant under the different conditions tested (presence or absence of A. polyphaga), hilA promoter activity is increased when the amoebae were present in close contact (Fig. 4B). Altogether, these results suggest that $A$. polyphaga induces hilA expression, which in turn probably activates the expression of SPI-1 genes. This suggests that resistance of $\mathrm{F} 113$ to predation requires contact between the two organisms.

\section{Conclusions}

Pseudomonas fluorescens $\mathrm{F} 113$ possesses two T3SSs belonging to the Hrp1 and SPI-1 families. The genetic expression of these two loci is regulated by the alternative sigma factor RspL and the transcriptional activator HilA respectively. While the role of Hrp1 is unknown, SPI-1 seems to be involved in grazing resistance to bacteriovorous protists. Identification and subsequent characterization of T3Es translocated by the SPI-1 T3SS will probably provide useful information about the mechanistic basis of F113 resistance to grazing.

\section{Acknowledgements}

This research was supported in parts by grants awarded to F. O'G. by the Science Foundation of Ireland (07IN.1/ B948, 08/RFP/GEN1295, 08/RFP/GEN1319, SFI09/RFP/ BMT2350); the Department of Agriculture, Fisheries and Food (RSF Grants 06-321 and 06-377; FIRM Grants 06RDC459 06RDC506 and 08RDC629); the European 
Commission (MTKD-CT-2006-042062, Marie Curie TOK: TRAMWAYS, EU256596, MicroB3-287589-OCEAN2012, MACUMBA-CP-TP 311975; PharmaSea-CP-TP 312184); IRCSET (05/EDIV/FP107/INTERPAM, EMBARK), the Marine Institute Beaufort award (C\&CRA 2007/082), the Environmental Protection Agency (EPA 2006-PhD-S-21, EPA 2008PhD-S-2) and the HRB (RP/2006/271, RP/2007/290, HRA/ 2009/146). Authors wish to thank Olivier Maillot and Magalie Barreau for technical assistance.

\section{References}

Aarons, S., Abbas, A., Adams, C., Fenton, A., and O'Gara, F. (2000) A regulatory RNA (PrrB RNA) modulates expression of secondary metabolite genes in Pseudomonas fluorescens F113. J Bacteriol 182: 3913-3919.

Abbas, A., Morrissey, J.P., Marquez, P.C., Sheehan, M.M., Delany, I.R., and O'Gara, F. (2002) Characterization of interactions between the transcriptional repressor PhIF and its binding site at the phIA promoter in Pseudomonas fluorescens F113. J Bacteriol 184: 3008-3016.

Abby, S.S., and Rocha, E.P.C. (2012) The non-flagellar type III secretion system evolved from the bacterial flagellum and diversified into host-cell adapted systems. PLoS Genet 8: e1002983.

Altier, C., Suyemoto, M., Ruiz, A.I., Burnham, K.D., and Maurer, R. (2000) Characterization of two novel regulatory genes affecting Salmonella invasion gene expression. Mol Microbiol 35: 635-646.

Bajaj, V., Hwang, C., and Lee, C.A. (1995) HilA is a novel OmpR/ToxR family member that activates the expression of Salmonella typhimurium invasion genes. Mol Microbiol 18: 715-727.

Barahona, E., Navazo, A., Yousef-Coronado, F., Aguirre de Carcer, D., Martinez-Granero, F., Espinosa-Urgel, M., et al. (2010) Efficient rhizosphere colonization by Pseudomonas fluorescens F113 mutants unable to form biofilms on abiotic surfaces. Environ Microbiol 12: 3185-3195.

Barahona, E., Navazo, A., Martinez-Granero, F., Zea-Bonilla, T., Perez-Jimenez, R.M., Martin, M., and Rivilla, R. (2011) Pseudomonas fluorescens $\mathrm{F} 113$ mutant with enhanced competitive colonization ability and improved biocontrol activity against fungal root pathogens. Appl Environ Microbiol 77: 5412-5419.

Barret, M., Egan, F., Fargier, E., Morrissey, J.P., and O'Gara, F. (2011) Genomic analysis of the type VI secretion systems in Pseudomonas spp.: novel clusters and putative effectors uncovered. Microbiology 157: 1726-1739.

Barret, M., Egan, F., and O'Gara, F. (2013) Distribution and diversity of bacterial secretion systems across metagenomic datasets. Environ Microbiol Rep 5: 117-126.

de Carcer, D.A., Martin, M., Mackova, M., Macek, T., Karlson, U., and Rivilla, R. (2007) The introduction of genetically modified microorganisms designed for rhizoremediation induces changes on native bacteria in the rhizosphere but not in the surrounding soil. ISME J 1: 215-223.

Chauhan, A., Layton, A.C., Williams, D.E., Smartt, A.E., Ripp, S., Karpinets, T.V., et al. (2011) Draft genome sequence of the polycyclic aromatic hydrocarbon-degrading, genetically engineered bioluminescent bioreporter Pseudomonas fluorescens HK44. J Bacteriol 193: 5009-5010.
Coombes, B.K. (2009) Type III secretion systems in symbiotic adaptation of pathogenic and non-pathogenic bacteria. Trends Microbiol 17: 89-94.

Cornelis, G.R. (2006) The type III secretion injectisome. Nat Rev Microbiol 4: 811-825.

Cornelis, G.R. (2010) The type III secretion injectisome, a complex nanomachine for intracellular 'toxin' delivery. Biol Chem 391: 745-751.

Correa, V.R., Majerczak, D.R., Ammar, E.-D., Merighi, M., Pratt, R.C., Hogenhout, S.A., et al. (2012) The bacterium Pantoea stewartii uses two different type III secretion systems to colonize its plant host and insect vector. Appl Environ Microbiol 78: 6327-6336.

Cronin, D., Moenne-Loccoz, Y., Fenton, A., Dunne, C., Dowling, D.N., and O'Gara, F. (1997a) Role of 2,4diacetylphloroglucinol in the interactions of the biocontrol pseudomonad strain F113 with the potato cyst nematode Globodera rostochiensis. Appl Environ Microbiol 63: 13571361.

Cronin, D., Moenne-Loccoz, T., Fenton, A., Dunne, C., Dowling, D.N., and Ogara, F. (1997b) Ecological interaction of a biocontrol Pseudomonas fluorescens strain producing 2,4-diacetylphloroglucinol with the soft rot potato pathogen Erwinia carotovora subsp. atroseptica. FEMS Microbiol Ecol 23: 95-106.

Cusano, A.M., Burlinson, P., Deveau, A., Vion, P., Uroz, S., Preston, G.M., and Frey-Klett, P. (2011) Pseudomonas fluorescens BBc6R8 type III secretion mutants no longer promote ectomycorrhizal symbiosis. Environ Microbiol Rep 3: 203-210.

De La Fuente, L., Landa, B.B., and Weller, D.M. (2006) Host crop affects rhizosphere colonization and competitiveness of 2,4-Diacetylphloroglucinol-producing Pseudomonas fluorescens. Phytopathology 96: 751-762.

Dean, P. (2011) Functional domains and motifs of bacterial type III effector proteins and their roles in infection. FEMS Microbiol Rev 35: 1100-1125.

Delany, I.R. (1999) Genetic analysis of the production of the antifungal metabolite 2,4-diacetylphloroglucinol by the biocontrol strain Pseudomonas fluorescens $\mathrm{F} 113$. PhD Thesis. Cork, Ireland: National University of Ireland.

Ehrbar, K., Friebel, A., Miller, S.I., and Hardt, W.D. (2003) Role of the Salmonella pathogenicity island 1 (SPI-1) protein InvB in type III secretion of SopE and SopE2, two Salmonella effector proteins encoded outside of SPI-1. J Bacteriol 185: 6950-6967.

Fenton, A.M., Stephens, P.M., Crowley, J., O'Callaghan, M., and O'Gara, F. (1992) Exploitation of gene(s) involved in 2,4-diacetylphloroglucinol biosynthesis to confer a new biocontrol capability to a Pseudomonas strain. Appl Environ Microbiol 58: 3873-3878.

Galan, J.E. (1999) Interaction of Salmonella with host cells through the centisome 63 type III secretion system. Curr Opin Microbiol 2: 46-50.

Hutcheson, S.W., Bretz, J., Sussan, T., Jin, S.M., and Pak, K. (2001) Enhancer-binding proteins HrpR and HrpS interact to regulate hrp-encoded type III protein secretion in Pseudomonas syringae strains. J Bacteriol 183: 5589-5598.

Huynh, T., Dahlbeck, D., and Staskawicz, B. (1989) Bacterial blight of soybean: regulation of a pathogen gene determining host cultivar specificity. Science 245: 1374-1377. 
Izoré, T., Job, V., and Dessen, A. (2011) Biogenesis, regulation, and targeting of the type III secretion system. Structure 19: 603-612.

Jackson, R.W., Preston, G.M., and Rainey, P.B. (2005) Genetic characterization of Pseudomonas fluorescens SBW25 rsp gene expression in the phytosphere and in vitro. J Bacteriol 187: 8477-8488.

Jousset, A., Rochat, L., Pechy-Tarr, M., Keel, C., Scheu, S., and Bonkowski, M. (2009) Predators promote defence of rhizosphere bacterial populations by selective feeding on non-toxic cheaters. ISME J 3: 666-674.

Jousset, A., Rochat, L., Scheu, S., Bonkowski, M., and Keel, C. (2010) Predator-prey chemical warfare determines the expression of biocontrol genes by rhizosphere-associated Pseudomonas fluorescens. Appl Environ Microbiol 76: 5263-5268.

Kimbrel, J.A., Givan, S.A., Halgren, A.B., Creason, A.L., Mills, D.I., Banowetz, G.M., et al. (2010) An improved, high-quality draft genome sequence of the GerminationArrest Factor-producing Pseudomonas fluorescens WH6. BMC Genomics 11: 522.

Kovach, M.E., Elzer, P.H., Hill, D.S., Robertson, G.T., Farris, M.A., Roop, R.M., and Peterson, K.M. (1995) Four new derivatives of the broad host-range vector pBBR1MCS, carrying different antibiotic resistance cassettes. Gene 166: 175-176.

Loper, J.E., Hassan, K.A., Mavrodi, D.V., Davis, E.W., II, Lim, C.K., Shaffer, B.T., et al. (2012) Comparative genomics of plant-associated Pseudomonas spp.: insights into diversity and inheritance of traits involved in multitrophic interactions. PLoS Genet 8: e1002784.

Lostroh, C.P., Bajaj, V., and Lee, C.A. (2000) The cis requirements for transcriptional activation by HilA, a virulence determinant encoded on SPI-1. Mol Microbiol 37: 300315.

Marguerettaz, M., Pieretti, I., Gayral, P., Puig, J., Brin, C., Cociancich, S., et al. (2011) Genomic and evolutionary features of the SPI-1 type III secretion system that is present in Xanthomonas albilineans but is not essential for xylem colonization and symptom development of sugarcane leaf scald. Mol Plant Microbe Interact 24: 246-259.

Martínez-Granero, F., Rivilla, R., and Martin, M. (2006) Rhizosphere selection of highly motile phenotypic variants of Pseudomonas fluorescens with enhanced competitive colonization ability. Appl Environ Microbiol 72: 3429-3434.

Matz, C., Nouri, B., McCarter, L., and Martinez-Urtaza, J. (2011) Acquired type III secretion system determines environmental fitness of epidemic Vibrio parahaemolyticus in the Interaction with bacterivorous protists. PIOS ONE 6: e20275.

Mavrodi, D.V., Loper, J.E., Paulsen, I.T., and Thomashow, L.S. (2009) Mobile genetic elements in the genome of the beneficial rhizobacterium Pseudomonas fluorescens Pf-5. BMC Microbiol 9: 8.

Mavrodi, D.V., Joe, A., Mavrodi, O.V., Hassan, K.A., Weller, D.M., Paulsen, I.T., et al. (2011) Structural and functional analysis of the type III secretion system from Pseudomonas fluorescens Q8r1-96. J Bacteriol 193: 177-189.

Mazurier, S., Lemunier, M., Siblot, S., Mougel, C., and Lemanceau, P. (2004) Distribution and diversity of type III secretion system-like genes in saprophytic and phy- topathogenic fluorescent pseudomonads. FEMS Microbiol Ecol 49: 455-467.

Miki, T., Iguchi, M., Akiba, K., Hosono, M., Sobue, T., Danbara, H., and Okada, N. (2010) Chromobacterium pathogenicity island 1 type III secretion system is a major virulence determinant for Chromobacterium violaceuminduced cell death in hepatocytes. Mol Microbiol 77: 855872.

Neidig, N., Paul, R.J., Scheu, S., and Jousset, A. (2011) Secondary metabolites of Pseudomonas fluorescens $\mathrm{CHAO}$ drive complex non-trophic interactions with bacterivorous nematodes. Microb Ecol 61: 853-859.

Ortet, P., Barakat, M., Lalaouna, D., Fochesato, S., Barbe, V., Vacherie, B., et al. (2011) Complete genome sequence of a beneficial plant root-associated bacterium, Pseudomonas brassicacearum. J Bacteriol 193: 3146.

Pallen, M.J., Beatson, S.A., and Bailey, C.M. (2005) Bioinformatics, genomics and evolution of non-flagellar type-III secretion systems: a Darwinian perpective. FEMS Microbiol Rev 29: 201-229.

Preston, G.M. (2007) Metropolitan microbes: type III secretion in multihost symbionts. Cell Host Microbe 2: 291-294.

Preston, G.M., Bertrand, N., and Rainey, P.B. (2001) Type III secretion in plant growth-promoting Pseudomonas fluorescens SBW25. Mol Microbiol 41: 999-1014.

Pukatzki, S., Kessin, R.H., and Mekalanos, J.J. (2002) The human pathogen Pseudomonas aeruginosa utilizes conserved virulence pathways to infect the social amoeba Dictyostelium discoideum. Proc Natl Acad Sci USA 99: 3159-3164.

Rainey, P.B. (1999) Adaptation of Pseudomonas fluorescens to the plant rhizosphere. Environ Microbiol 1: 243-257.

Redondo-Nieto, M., Barret, M., Morrisey, J.P., Germaine, K., Martínez-Granero, F., Barahona, E., et al. (2012) Genome sequence of the biocontrol strain Pseudomonas fluorescens F113. J Bacteriol 194: 1273-1274.

Rezzonico, F., Defago, G., and Moenne-Loccoz, Y. (2004) Comparison of ATPase-encoding type III secretion system hrcN genes in biocontrol fluorescent Pseudomonads and in phytopathogenic proteobacteria. Appl Environ Microbiol 70: 5119-5131.

Rezzonico, F., Binder, C., Defago, G., and Moenne-Loccoz, Y. (2005) The type III secretion system of biocontrol Pseudomonas fluorescens KD targets the phytopathogenic chromista Pythium ultimum and promotes cucumber protection. Mol Plant Microbe Interact 18: 991-1001.

Rietsch, A., and Mekalanos, J.J. (2006) Metabolic regulation of type III secretion gene expression in Pseudomonas aeruginosa. Mol Microbiol 59: 807-820.

Rosenberg, K., Bertaux, J., Krome, K., Hartmann, A., Scheu, S., and Bonkowski, M. (2009) Soil amoebae rapidly change bacterial community composition in the rhizosphere of Arabidopsis thaliana. ISME J 3: 675-684.

Sait, M., Kamneva, O.K., Fay, D.S., Kirienko, N.V., Polek, J., Shirasu-Hiza, M.M., and Ward, N.L. (2011) Genomic and experimental evidence suggests that Verrucomicrobium spinosum interacts with eukaryotes. Front Microbiol 2: 211.

Shanahan, P., O'Sullivan, D.J., Simpson, P., Glennon, J.D., and O'Gara, F. (1992) Isolation of 2,4diacetylphloroglucinol from a fluorescent pseudomonad 
and investigation of physiological parameters influencing its production. Appl Environ Microbiol 58: 353-358.

Sheikh, J., Dudley, E.G., Sui, B.Q., Tamboura, B., Suleman, A., and Nataro, J.P. (2006) EilA, a HilA-like regulator in enteroaggregative Escherichia coli. Mol Microbiol 61: 338350.

Sperandio, D., Decoin, V., Latour, X., Mijouin, L., Hillion, M., Feuilloley, M., et al. (2012) Virulence of the Pseudomonas fluorescens clinical strain MFN1032 towards Dictyostelium discoideum and macrophages in relation with type III secretion system. BMC Microbiol 12: 223.

Tan, M.-W., Mahajan-Miklos, S., and Ausubel, F.M. (1999) Killing of Caenorhabditis elegans by Pseudomonas aeruginosa used to model mammalian bacterial pathogenesis. Proc Natl Acad Sci USA 96: 715-720.

Tang, X.Y., Xiao, Y.M., and Zhou, J.M. (2006) Regulation of the type III secretion system in phytopathogenic bacteria. Mol Plant Microbe Interact 19: 1159-1166.

Troisfontaines, P., and Cornelis, G.R. (2005) Type III secretion: more systems than you think. Physiology 20: 326339.

Villacieros, M., Power, B., Sánchez-Contreras, M., Lloret, J., Oruezabal, R.I., Martín, M., et al. (2003) Colonization behaviour of Pseudomonas fluorescens and Sinorhizobium meliloti in the alfalfa (Medicago sativa) rhizosphere. Plant Soil 251: 47-54.

Viollet, A., Corberand, T., Mougel, C., Robin, A., Lemanceau, P., and Mazurier, S. (2011) Fluorescent pseudomonads harboring type III secretion genes are enriched in the mycorrhizosphere of Medicago truncatula. FEMS Microbiol Ecol 75: 457-467.

Wu, W., and Jin, S. (2005) PtrB of Pseudomonas aeruginosa suppresses the type III secretion system under the stress of DNA damage. J Bacteriol 187: 6058-6068.

Xu, Z., and Hao, B. (2009) CVTree update: a newly designed phylogenetic study platform using composition vectors and whole genomes. Nucleic Acids Res 37: W174-W178.

Zwiesler-Vollick, J., Plovanich-Jones, A.E., Nomura, K., Bandyopadhyay, S., Joardar, V., Kunkel, B.N., and He, S.Y. (2002) Identification of novel hrp-regulated genes through functional genomic analysis of the Pseudomonas syringae pv. tomato DC3000 genome. Mol Microbiol 45: 12071218.

\section{Supporting information}

Additional Supporting Information may be found in the online version of this article at the publisher's web-site:

Appendix S1. Experimental procedures

Table S1. Transcriptome profiles of pBBR1MCS-hilA and pBBR1MCS-rspL strains.
Table S2. Prediction of RspL binding site in the genome of P. fluorescens $\mathrm{F} 113$.

Table S3. Bacterial strains and plasmids used.

Table S4. Oligonucleotides used.

Fig. S1. Distribution of T3SSs in Pseudomonas spp. Phylogenomic analysis of 135 Pseudomonas genomes was performed using CVTree (Xu and Hao, 2009). Escherichia coli $\mathrm{K} 12-\mathrm{DH} 10 \mathrm{~B}$ was used as out-group. Strains highlighted in brown are related to the $P$. fluorescens group. Genomes related to $P$. protegens Pf-5, P. fluorescens Pf0-1, P. fluorescens SBW25, $P$. brassicacearum NFM421 and $P$. fluorescens have been sequence entirely, while genomes of strains belonging to the $P$. fluorescens group are incomplete. The ORFs (open reading frames) PA1703, PSPTO_1402, PSPPH_2520 and PSF113_1781 (COG4789), representing archetypical Ysc, Hrp1, Rhizobial and SPI-1 T3SS proteins, were used as baits in sequential BLASTp and TBLASTn searches to identify T3SS loci in the 135 Pseudomonas genomic sequences. Square dots indicate that the T3SS-loci are incomplete.

Fig. S2. Genes regulated by RspL and HilA.

$A$ and $B$. Comparisons of transcripts abundance between F113 carrying pBBR1MCS5-rspL or pBBR1MCS5-hilA (+) and F113 carrying the empty vector pBBR1MCS5 (-).

C. Plot of F113 pBBR1MCS5-rspL versus F113 pBBR1MCS5 average log intensities.

D. Plot of F113 pBBR1MCS5-hilA versus F113 pBBR1MCS5 average log intensities.

E. Venn diagram showing the overlap between genes differentially regulated in pBBR1MCS5-hilA and pBBR1MCS5-rspL.

Fig. S3. Promoter fusion activities of $r s p L$ and hilA in LB NTA medium. Strains of $P$. fluorescens $\mathrm{F} 113$ carrying $r s p L$ and hilA transcriptional fusions were grown in LB NTA $(10 \mathrm{mM})$ at $30^{\circ} \mathrm{C}$ shaking. Growth curves (principal $y$ axis) and relative fluorescens units (secondary $y$ axis) were monitored in F113 pPROBE-GT (blue diamond), F113 pPROBE-rspL (red square), F113 pPROBE-hilA (green triangle), in FG9 pPROBE-rspL (purple arrow) and FG9 pPROBE-hilA (black arrow). Each value is the mean of three samples obtained from the same biological replicate.

Fig. S4. P. fluorescens virulence towards $C$. elegans worms. Slow killing Kaplan-Meier survival plots of worms fed with P. fluorescens F113 wt $(n=113), \Delta r s c U(n=228), \Delta r s c U$ pBBR1-rscU $(n=124), \Delta$ spaS $(n=158)$ or $\Delta$ spaS pBBR1spaS $(n=173)$. Each value reported for the assay is the mean of measurements of six samples from two independent experiments. Asterisks indicate significant differences $(P<0.001)$ between $\mathrm{F} 113$ wild type and the corresponding mutant tested, as assessed by pairwise comparisons (log rank test). The strain FG9 is a F113 derivative carrying a transposon insertion in gacA (Delany, 1999). 